\title{
Der St. Gotthard - Bedeutung und Auswirkungen
}

Der Schnittpunkt der Nord-Süd-Linie über den Gotthard mit der inneralpinen Längslinie vom Lac Léman zum Bodensee ist das Zentrum unseres Staates, geologisch, morphologisch, verkehrspolitisch und kulturell.

Paul Niggli 1940

Bereits seit Generationen läuft die breitgespannt interdisziplinäre, immer wieder fruchtbar angeregte Diskussion um die Zeitfrage des Eintritts der Gotthardwildnis in die Kulturgeschichte. Dabei war eigentlich immer zu bedenken, daß es zwei Möglichkeiten der «Eröffnung» des Passes gab: einerseits seine Benützung im engeren Sinne, d. h. die Überschreitung des Gotthardmassivs, und anderseits jene zusätzliche Querung des Aarmassivs, was die spektakuläre Öffnung der europäischen N-S-Transversale bedeutet. Auf diese prinzipielle Dualität im Paßbegriff hat Carl Ritter (1779-1859) in seinen Berliner Vorlesungen «Europa» hingewiesen, die posthum 1863 von seinem Schüler Dr. H. A. Daniel auf Grund des Ordinarius eigenen «Collegienheften» herausgegeben wurden. Den wertvollen Band einführend, ehrt der Schüler den großen Lehrer: «Welche nie nachlassende Sorgfalt, nach Inhalt und Form das, was er mitteilte, immer vollendeter zu gestalten!» Für Ritter waren zwei Schweizer Aufenthalte - 1807 Yverdon mit Pestalozzi und seinem Geographen J. G. G. Tobler, 1811/12 im geist- und kunsterfüllten Genf - prägend geworden. Umgekehrt saßen später in seinen Berliner Vorlesungen immer wieder auch junge Schweizer, vor allem Zürcher, von Arnold Escher von der Linth bis Hans Georg Nägeli. Wo Ritter die vertikale Gliederung des Kontinentes darstellt, kommt im dicht konzipierten Abschnitt «Europas Plastik und die Wissenschaft» der frühe und wissenschaftsgeschichtlich (z. B. im Blick auf Alpenforschung) fast prophetische Länderkundler zum Ausdruck. Und nun also lesen wir im Kapitel «Alpenpässe» nach vorangehender Unterscheidung zwischen Längstal- und Quertalpässen: «Wir machen fernerweit einen Unterschied zwischen Alpenpassage und Alpenpaß. Die Alpenpässe sind die höchsten Einschnitte des Hochgebirges, öfter nur wenige Stunden lang, zuweilen nur $1 / 4$ Stunde breit. Sie liegen meist in unwirthbarer Wildnis voll Felsentrümmer, Eis- und Schneemassen. Zur Aufnahme der Reisenden sind die Hospize
(Klöster, Tavernen, Tauernhäuser) errichtet. Alpenpassagen nennen wir alle Alpenübergänge, die aus der Ebene über das Gebirge wieder bis zur nächsten Ebene hinüberführen, z. B. die Mont-Cenis-Straße, die Gotthardstraße, die Brennerstraße. Die Länge derselben ist bedeutender (6-10-20 Stunden).» Die konsequente Beachtung dieser (scheinbar lapidaren) Ritterschen Definition hätte gelegentlich in der Gotthardforschung nützlich sein können.

Wer sich bemüht, in die Frage um die Erschließung des Gotthardes oder speziell der Schöllenen ernstlich Einblick zu erhalten, wird Mitbeobachter eines über Jahrzehnte anhaltenden Einsatzes historischer und geographischer Forschung. Der Kampf um Abklärung beeindruckt durch seine grundsätzliche, oft akribisch geführte Belegungs- und Widerlegungssuche. Und diese Diskussion zeigt allein schon, daß Eröffnungsfrage und geographischer Gegenstand sich decken in ihrer außergewöhnlichen Bedeutung.

Es kann durchaus nützlich sein, gar nicht eilends meridional den $\mathrm{Pa} \beta$ anzustreben, sondern primär davon auszugehen, dass Ursern (Ursaria) zentral in der markanten alpentektonischen W-O-Furche liegt, welche (ca. 25 v. Chr.) im «gewaltigen Zusammenhang augusteischer Politik» (Gaglardi 1938) erschlossen wurde und zur Verbindung Valesia-Raetia trieb. Damit erscheint «Welt» im Hochtal der «statio Ursariorum». Es ist keineswegs das erste menschliche Leben, das damit hier zum Ausdruck kommt. 1955 wurde bei Arbeiten an der Gotthardstraße im Mätteli oberhalb Hospental eine neolithische Silex-Pfeilspitze gefunden. Das Artefakt belegt also nebenbei frühe (ca. 4000 v. Chr.) nomadische Jagdkultur. Aber wir haben das römische Aufblühen der Transversale St. Maurice-Chur anvisiert. Alexander Tanner hat 1963-68 mit Grabungen bei Truns ein außerordentlich reichhaltiges Fundmaterial aus Ur- und Frühzeit und Mittelalter zutage gebracht (helvetia archaeologica 1970/3). Er erklärt die überraschende Fülle u. a. mit der «geographischen resp. verkehrsgeographischen Lage der Örtlichkeit»: nur $10 \mathrm{~km}$ vor der Straßenteilung in Disentis zum «gut begehbaren Lukmanier» nach Sü-

Emil Egli, Dr. phil., a. Prof., Hochstraße 49, 8044 Zürich 
den und nach Westen zum Oberalp und weiter über die Furka ins Wallis. «Die Verbindung Graubünden-Wallis muß schon sehr früh bestanden haben. Durch einen Grabstein eines hohen römischen Beamten aus augusteischer Zeit wissen wir, da $\beta$ damals Rätien und Wallis eine einzige Provinz bildeten, wozu sicher das Bestehen einer Straße Voraussetzung war.»

Damit also liegt das Hochtal Ursern im Längskorridor der Alpen, der sich der Geschichtsschreibung gelegentlich als Rochadelinie einprägen wird. Ursern ist darin doppelseitig aufgeschlossen durch die Tore der Oberalp und der Furka. $\mathrm{Da}$ die Paßlücke des Gotthards - eiszeitlich durch die Tansfluenz des Reußgletschers geweitet und kräftiger als der Julier glazial eingetieft - zur Verkehrsergänzung über die Hauptwasserscheide lockte, ist klar. Sie weckte Kulturtransfluenz. Da sprechen auch die zahlreichen römischen Funde in der Leventina und Riviera mit, soweit sie nicht $z$. T. dem San Bernardino und Lukmanier zuzuordnen sind. Mit der Pässetrilogie Oberalp - Furka - Gotthard hat auch der letztere fraglos seine frühe Phase der Bedeutung, Bedeutung des «Alpenpasses», aber noch nicht der «Alpenpassage» nach Ritterscher Konsequenz.

Ursern hat durchaus seine oft genannten römischen Münzen. Da ist z. B. der im Garten des Hotels Meyerhof zu Hospental gefundene Denar von Licinianus (Kaiser von 307 bis 323). Sie lag also auf dem Talboden im Schnittpunkt des großen (westöstlichen und nord-südlichen) Gotthardstraßenkreuzes. Dann ist da eine andere, für die Diskussion um das historische "Alter» des Passes nie außer acht gelassene Münze: ein Doppeldenar von Trebonianus Gallus (Kaiser von 252 bis 254). Immer wieder Anstoß zu ihrer Deutung erregte vor allem der Fundort: Sie ist mehr als $600 \mathrm{~m}$ über dem Ursner Talboden gehoben worden, auf der markanten Alpterrasse des Bäzberghangs, Höhe von Roßmettlen, $2100 \mathrm{~m}$ ü. M., nahe bei Roßplatten. Auf die Münze ist zurückzukommen.

Kardinale Fragen drängen sich heran. (Es ist weder der Raum noch der Zweck dieses Beitrages, eine schrittweise Gotthardgeschichte zu schreiben. Es geht um einige kulturgeographische Zusammenhänge und zeitgeistbedingte Entwicklungssprünge; deren einer schließlich die harte EG-Gotthardaktualität ist.) - Römische Münzstreufunde? Ja. Aber wo sind am zentralsten und kürzesten Alpenpaß die imponierenden römischen Straßenbauten, wie sie an andern Pässen übermittelt und vor allem durch Armon Planta (1976) an der Julierroute erforscht worden sind? Da zeigt sich die vollendete Kunst des römischen Kulturingenieurs, Straßen geländeharmonisch in die Landschaft zu legen. Die heutige Autostraße z. B. folgt dem Silvaplanersee, um dann mit weitausholenden Kehren die Mündungsstufe Valluntobel - des Juliertales zu überwinden. Die Römerstraße aber ist in den Berghang gehoben und erreicht «gleichmäßig ansteigend» den oberen Rand der $200 \mathrm{~m}$ hohen Vallunstufe. Von dort folgt sie gleichermaßen bequem dem langen Taltrog zum $\mathrm{Pa}$. Armon Planta hat Fahrrinnen von bis $40 \mathrm{~cm}$ Tiefe freigelegt, «die erkennen lassen, wie fein die mindestens $140 \mathrm{~cm}$ hohen Räder waren. Ihre Reifen waren nur $4 \mathrm{~cm}$ breit. Radabstand wie in allen andern Orten $107 \mathrm{~cm}$ ». Am Maloja-Absturz auf die oberste Talstufe des Bergells findet sich am rechtsseitigen Talhang ein «Paradestück des römischen Fahrwegs»: In eine $8 \mathrm{~m}$ lange Felsplatte sind «zwischen die Radrinnen 14 Tritte für das Zugtier in den Fels gehauen». Wo liegen an Bäzberg, Tremola, Piottino entsprechende Beweisstücke für römische "Alpenpassage» am Gotthard, wo sich die Taten bereits europäisch triumphal und «bezahlt» gemacht hätten?

Da einerseits die Münzfunde in Ursern ihre wichtige Rolle spielen, anderseits die Rittersche Paßdefinition Klarheit schafft, ist weiter ein Satz in August Pünteners «Urner Münz- und Geldgeschichte» (1980) zu beachten: «Nach dem Zerfall des Römischen Reiches ist aus Uri wohl ein geldwirtschaftliches Niemandsland geworden. Aus der Zeit der Merowinger und Karolinger (5.-9. Jh.), die das Münzwesen erneuerten und belebten, sind in Uri keine Münzfunde bekannt.» In das Phänomen des Kulturstromes am Gotthard drängt sich der Forschung immer wieder die Irritation von dessen Retardierung auf (im Vergleich vor allem mit der Geschichte der Bündner Pässe).

Die Gotthardgeschichte wurde nicht nur am $\mathrm{Pa}$, sondern fast akribischer noch in und an der Schöllenen geschrieben. $\mathrm{Pa} \beta$ und Riegel sind in der Gebirgsgeographie zwei hervortretende Landschaftselemente, die kulturgeschichtlich mitreden. Da ist also der Schöllenenriegel. Karl Meyer (1912/1919 und im Sammelband 1952), nach kurzer Darstellung der alten, grundsätzlichen Verschiedenheit Ursern / Uri, schreibt: "Diese wirtschaftliche, politische und kirchliche Zweiteilung des Reußtales beweist, $\mathrm{da} B$ in der Entstehungszeit dieser Verhältnisse, im frühen und im hohen Mittelalter, eine richtige Verbindung zwischen Uri und Ursern noch fehlte. Auch andere Indizien sprechen gegen das Vorhandensein eines praktikabeln Gotthardweges.» Die Felsbarriere, an der engsten Stelle des Chilchbergs gelegentlich einfach auch «Stein» genannt, wie Bergünerstein, Oberhalbstein usw., greift tief durch die Humangeographie, so daß sich Felix Aschwanden und Walter Clauss in ihrem großen «Urner Mundart Wörterbuch» (1982) «eine besondere Behandlung der Mundart des Urserentales aufdrängt». Weder das «Ewige Landrecht, das im Jahre 1410 zwischen Uri und Ursern geschlossen wurde», noch die anschwellende Mobilität reichten bis anhin aus, «um einen spürbaren Ausgleich zwischen den Dialekten „ob" und „nid" der Schöllenen zu erstellen». Es folgen zahlreiche Belege. Und da ist also das Tal mit den 
drei Paßtoren. Durch sie kommen die frühen romanischen und später die walserischen Siedler: die Danioth (Danioti in Venedig) und Russi - die Meyer und Renner von Osten - die Christen und Nager von Westen. Danioth in «Die Ursener Geschlechter» (1978) läßt die Namenreihe voll erklingen und fügt bei: «Kein einziges der alten Geschlechter stammt nachweisbar aus dem alten Kantonsteil Uri.» Und Karl Peter, in seiner einfühlenden Darstellung «Der Urschner» (1978), gibt folgenden, im Blick auf die Leventina nicht unwichtigen Hinweis: «Schon die alten Urschner Bauern verstanden sich durch ihre wirtschaftliche Ausrichtung nach dem Süden in verblüffender Weise auf das typisch milanesische Italiänisch.» Bei der erstmaligen urkundlichen Erwähnung 1203 gehört Ursern zum weiten Grundbesitz der Benediktinerabtei Disentis, gegründet um 750 , d. h. mit einer ersten Zelle um 700 an der Paßgabelung Lukmanier / Oberalp. Im Jahre 1649 kauften sich die Ursner von den Lehenzinsen los.

In diesem so besondern, zentralalpin tektonisch verklemmten Hochtal mit der Pässetrilogie entsteht durch die gemeinschaftsbildende Überblickbarkeit und zunächst ausschließliche Alpwirtschaft eine deutliche Talgenossame - und der unverwechselbare Ursner. Dem hochgeschobenen Rand der Ökumene entspricht die angepaßte alpwirtschaftliche Organisation: «Im ganzen Gebiet der Korporation Ursern gibt es keine Privatalpen. Alle Weiden sind Allmende. Sogar die Wiesen des Talbodens sind nur beschränktes Privateigentum, denn nach altem Rechte werden sie ab 28. September, morgens $6 \mathrm{Uhr}$, bis Allerheiligen (1. November) für den Weidgang Allgemeingut» (Max Oechslin 1941). Über allem steht die korporative Talgenossenschaft. (Der Begriff Markgenossenschaft, wie er weitreichend benützt wird, ist sprachhistorisch eine "gewünschte Verbindung zum frühen Mittelalter» (...) «ist jedoch nicht nur nicht verwendet worden für die Talschaften der Innerschweiz, sondern er ist auch sachlich unzutreffend» (Paul Kläui 1964). Während in Uri die Lands- (resp. Landes-)gemeinde 1929 zugunsten der bloßen (kleineren) Korporationsgemeinde fallengelassen wurde, lebt in Ursern die Talgemeinde mit Talammann, Talarchivar, Talschreiber, Talkanzlei und verbindet die Generationen vital. Neben den jährlichen Korporationsproblemen kommen auch gemeindepolitische Vorhaben im Ring zu (durchaus jugendbeteiligter) Diskussion und Abstimmung: Gründung eines Talmuseums, Umgestaltung des Spittels von Andermatt (es gibt keine armen Wanderer mehr) in ein «Betagtenheim», eine vergandete Alp auf Gruschen soll wieder bestoßungsfähig gemacht werden; alle drei (auch finanziell) schwerwiegenden Anliegen an der Talgemeinde 1987 vorgelegt und beschlossen. Ein kleiner bergbeengter Lebensraum, durch den zwei weltlastige Straßen gehn, bewahrt seine genos- senschaftliche Identität. Wann aber wurde die nordsüdliche geomorphologische Furche Kulturkanal? Das heißt: Wann hat der Homo faber den sperrenden Felsriegel gemeistert? Es geht dabei um Dateneinengung. Da ist die «Alpenpaßpolitik Friedrich Barbarossas» im 12. Jahrhundert. Es handelt sich um Sicherung der Südzugänge, und in seinem regen Hin und her über die Alpen benützt er fast alle, vor allem auch die Bündner Pässe, vom Brenner bis zum Mont Cenis - nie den Gotthard (H. Büttner, 1970). Aber immer wieder, wie an allen Paßschluchten, war Diskussion lebendig über die hohen seitlichen oder ausgreifenden Umgehungswege der Schöllenen. Der Chrüzlipaß, der sowohl zum Oberalp als vor allem zum Lukmanier führt, ist am eingehendsten (auch im Hinblick auf frühe Säumerei und Susten) erforscht von Max Oechslin (1928), Marcel Beck (1949), Alois Kocher (1951) und übereinstimmend als sehr wohl praktizierbarer «Vorläufer des St. Gotthardpasses» gewertet. Als der Lukmanier nach Öffnung der Schöllenen an Bedeutung verlor, sank auch das Interesse am Chrüzlipaß. Aber die Überlegungen konzentrierten sich vor allem auch auf die schluchtnahen seitlichen "Höhenwege». Wer sie begangen hat auch an heiklen Stellen, z. T. zusammen mit dem Sohn, ist gerne überzeugt, dass einheimische Jäger und Hirten trittsicher genug waren in Granit und Gneis des Aarmassivs. Einzelgänger waren wohl durch alle Jahrhunderte «oben üs» unterwegs, kaum aber Pilgergruppen, die sich in Disentis sammelten. Es geht uns um die historische Schnittstelle zwischen sporadisch begangener zweiter Wasserscheide und dem Einsatz des permanenten Kulturkorsos an der nord-südlichen Alpenpassage des Gotthards.

Einen kühnen theoretischen Versuch zur Vorverlegung großer Gotthardbenützung (gleichsam über Barbarossas Vorsicht hinweg?) machte Jakob Escher-Bürkli ( «Von der alten Gotthardstraße», 1935), indem er König Heinrich II. im Frühjahr 1004 die Rückreise aus Italien (als Möglichkeit, die Route ist nicht dokumentiert) über den Gotthard wählen läßt. Mit erstaunlicher örtlicher Recherchierung führt er ihn über den Bäzberg (die erwähnte römische Münze spielt nun eine Rolle) durch das steile Couloir der «Geißkehle» (heute total verwachsen, unmittelbar neben dem Abluftkamin des Straßentunnels) in die Schlucht hinunter. Als zweite Möglichkeit prüft er den Spitzigrat mit der «Leiden Kehle». Neben den topographischen Schwierigkeiten handelt es sich in den zwei Fällen um Gegensteigungen von mindestens 600 , maximal 1000 Meter. Escher-Bürkli kommt zum Schluß, «daß die Strecke Varese-Zürich nur über den Gotthard in 5 Tagen zurückgelegt werden konnte». Leonhard von Muralt rezensiert den Vortrag einige Tage später in der NZZ zurückhaltend. Karl Meyer (1912/1952) läßt Kaiser Heinrich II. der Lukmanier-Walensee-Route folgen, «denn seine Reiseetappe Lacunauara, bis- 


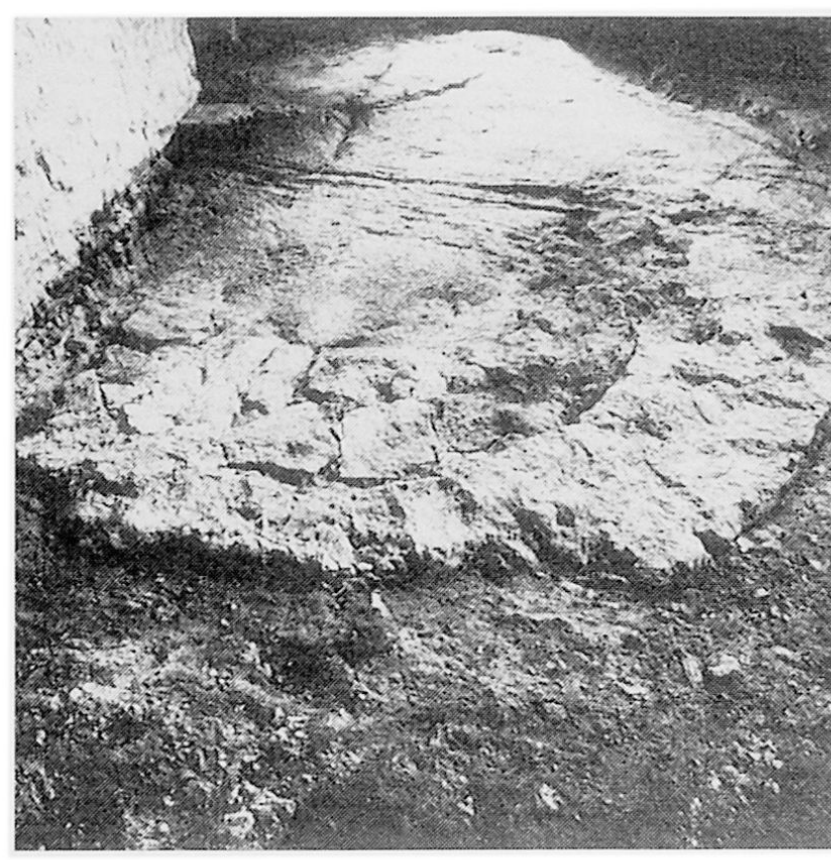

Abb. 1 a Fundamente der vorromanischen Apsis

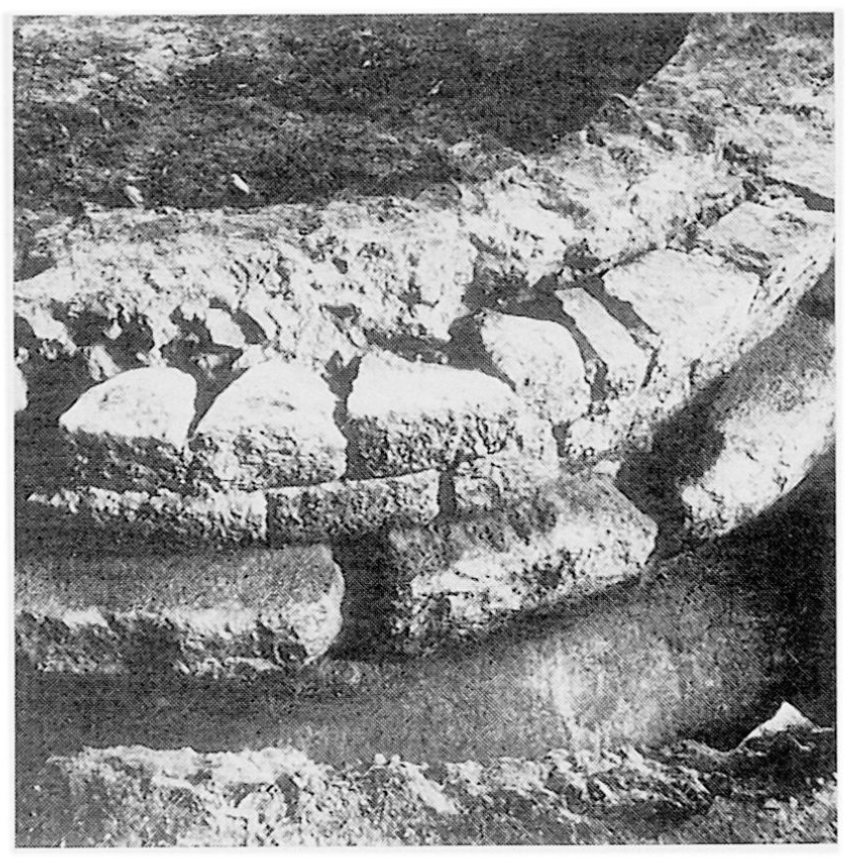

Abb. $1 \mathrm{~b}$ Fundamente der romanischen Apsis

Mit freundlicher Erlaubnis aus Mario Fransioli "Der St. Gotthard und seine Hospize», Gesellschaft für Schweizerische Kunstgeschichte, Bern, 1987.

her stets als Locarno gedeutet, ist identisch mit dem Walensee (Lacus ualaha)». Wer Gerold Meyer von Knonaus Schilderung «Ein kaiserlicher Kriegszug über einen Alpenpaß» liest (SAC-Jahrbuch 1878/79, 26 Seiten), wird vorsichtig mit Höhenwegvisionen. Neue fruchtbare Diskussion entstand, als 1975 bei Restaurationsarbeiten an der Kapelle auf der Paßkulmination unter deren Fußboden Fundamente einer romanischen und einer vorromanischen Apsis auf anstehendem Rundhöckerfels zum Vorschein kamen. (Darüber, wie über die Paßgeschichte, orientiert in ausgezeichnet dokumentierter Dichte die Schrift: Mario Fransioli «Der St. Gotthard und seine Hospize», mit Einführung von Denkmalpfleger P. A. Donati, Schweizerischer Kunstführer, Bern 1982.) Selbst die spärlichen Fundamentreste erlauben Fransioli überzeugende Bauvergleiche mit kleinen Kirchen im Tessin. Die romanische Kapelle (die genaue Bauzeit der vorromanisch-spätkarolingischen ist vorläufig nicht bekannt) wird geweiht zwischen 1166 und 1176 durch den Mailänder Erzbischof St. Galdinus. Passübergreifendes Interesse zur Linie St. Maurice-Chur mag mitbeteiligt gewesen sein. Aber da war noch ein anderes wartendes Bedürfnis, das zu wenig beachtet ist. Im ganzen Alpengebiet sind vielerorts alpwirtschaftliche Übergriffe der Hirten von den südlichen Paßflanken auf die Weiden der nordexponierten Hänge rechtlich praktiziert. Früh benützten die Leventiner Hirten die Alpen Isenmann, Wytenwassern und Gaggiola, ob Hospental und Realp. Diese Weiden wurden von den Ursnern schließlich 1331 vertraglich wieder zurückgenommen. (Otto Flückiger «Pässe und Gren- zen», 1928). Daß für die Hirten auf dem $\mathrm{Pa} ß$ und an seinen beidseitigen Flanken gottesdienstliche $\mathrm{Be}$ treuung gesichert sein wollte, steht außer Frage. Noch Hans Rudolf Schinz, 1783, betont die primäre Bedeutung der Kapelle für die Hirten neben der zusätzlichen Wanderhilfe.

Die üblich genannten Daten zur großen Paßeröffnung: 1218 verpfändet Friedrich II. die Vogtei Uri an die Habsburger. 1230: Der Erzbischof Enrico Settala weiht die Kapelle auf der Paßhöhe auf den Namen des heiligen Gotthard. 1231: König Heinrich VII., Sohn Friedrich II., gewährt Uri die Reichsfreiheit und entzieht damit das Gotthardtal dem Machtbereich der Grafen von Habsburg. 1236: "Erste historisch streng verbürgte Überschreitung» des Passes durch den Benediktinerabt Albert von Stade aus Bremen (C. J. Burckhardt). Örtlich zuverlässige Schilderung seiner Rückreise von Rom. 1237 Erste Erwähnung des Hospizes. Die «Straßenstatute von Osco» (5. April 1237) melden bereits einen bedeutenden Warenverkehr und den Übergang vieler dänischer Mönche über den $P a ß$.

Ins «erste Drittel des 13. Jahrhunderts» oder auf 1225 oder 1226 wird heute durchgehend die große Tat von europäischer Bedeutung am Schöllenenriegel angesetzt. Das am Gothard eine eigentliche Zeitwende bewirkende Geschehen liegt nicht mehr in legendärer Ungewißheit. Deutlich ist nun den Walsern das Werkzeug und zugleich das Können in die Hand gelegt. Sie erscheinen im 12. Jahrhundert über die Furka in Ursern (Paul Zinsli 1967 und neu 1982) mit der langen Erfahrung des kühnen Wasserleitungsbaus mittels Holz am senkrechten Fels. Da 
sind nun die zwei (zu lange in Verwirrung gehaltenen) jetzt nicht mehr zu verwechselnden Brücken: die «Twärrenbrücke», der Steg über der Reuß längs dem Kirchbergsporn, im Ursner Sprachgebrauch gewohnt und offenbar immer so bezeichnet, und die «Stiebende Brücke», der spätere Pons diaboli (wohl nach 1595, da sie als Steinbau erwähnt wird und die gelehrten Reisenden erscheinen). Es ist die Brücke mit dem «Staubregen, von dem man bedeckt wird» (Johann Georg Sulzer 1776 und entsprechend oder mit «durchnäßt» formuliert in ungezählten Tagebüchern). Die Teufelsbrücke «findet die erste Erwähnung im Habsburger Urbar (1303-1308). Sie wird dort «stiebende Brücke» geheißen (I. Meyer 1938, A. Kocher 1961). Ganz gleich verhält es sich mit dem sog. «Pfaffenbrief» von 1370. Es handelt sich um ein Übereinkommen zwischen den Waldstätten, Zug, Luzern und Zürich betreffend persönliche und richterliche Sicherheit (ausgelöst durch eine Gewalttat der Söhne des Zürcher Bürgermeisters Rudolf Brun). Da heißt es: «Sie gewähreten, daß von der stäubenden Brücke bis nach Zürich alle Straßen gegen alle Seiten ihres Landes jedem offen und sicher sein sollen...» Und der historisch behandelnde Johannes von Müller (Ausgabe 1833) merkt dazu an, daß «die Teufelsbrücke ... malerisch genug die stäubende Brücke genannt» werde. Das «Alte Stiftbuch» im Pfarrarchiv Andermatt enthält die Notiz: «Im Jar do man zaltt noch der geburt Jesu Christj Tusend Fünfundert Nünzig und im fünften Jahre ward die Steüben Brug in der Schelenen gebuwen", es handelt sich um den steinernen Neubau. Möglicherweise war der Teufelsname noch gar nicht heimisch. Eine Namenkonfusion besteht nicht mehr.

Die zwei Brücken haben nicht nur die Schöllenen als Schlucht passierbar gemacht, sie haben in europäischem Ausblick die nord-südliche Gotthardtransversale geöffnet. Rasch weitet sich die regionale Säumerei räumlich und organisatorisch aus, «modernisiert» sich mit Pflicht zu Seuchenmeldungen von Tal zu Tal. Die neue Verkehrssituation bewegt auch die Politik. «Die Schwyzer, die schon seit längerem darauf sannen, zu anerkannter Reichsfreiheit zu kommen, wie das 1231 den Urnern dank Heinrich (VII.) gelungen war [...] schickten eine Gesandtschaft nach Faenza, das Kaiser Friedrich II. seit September 1240 belagerte. Da für ihn ein neuer Parteigänger, zudem aus der Innerschweiz, höchst erwünscht war, erteilte er den Schwyzern den erbetenen Freiheitsbrief, obwohl die Schwyzer sich in einer schlechteren Ausgangslage befanden als die Urner, die offensichtlich an die Habsburger verpfändet gewesen waren und 1231 einfach wieder ausgelöst wurden, wobei sie die Pfandsumme wohl aus eigener Kraft dem König zur Verfügung stellten. Dazu waren sie in der Lage, da ihnen aus dem sich seit etwa 1200 lebhaft entwickelnden Gotthardverkehr stets flüssige Mittel zuströmten. [...] Damit sind wir nun wohl beim wichtigsten Motiv angelangt, das die Kaiser und deutschen Könige bewogen hat, den Talschaften an der Gotthardroute die Reichsfreiheit zu verleihen. Es lag ihnen sehr viel daran, an diesem für sie lebenswichtigen Übergang treue und zufriedene Gefolgsleute zu besitzen, die sich für den Unterhalt und die Offenhaltung der Pässe wirklich einsetzten» (Prof. Dietrich Schwarz, aus Vortragsmanuskript vom 22. Nov. 1990).* Der offene und machtumlauerte $\mathrm{Pa} \beta$ machte nicht nur Schweizer Geschichte, sondern auch europäische mit der "Anerkennung unserer Neutralität durch die kriegsmüden Großmächte im "Gnadenjahr" 1815» (Georg Thürer, 1944).

Bald hat der neue Gotthardweg seine Fernwirkung. Zürich wendet seinen Südverkehr zum Teil ab von der Walenseeroute nach Horgen, wo «spätestens 1452 eine Sust entstand», neben der Haab (Christian Renfer, 1982); zu Land geht es zum Zugersee und nochmals ein Stück Landweg bis Küßnacht zum Wasseranschluß nach Flüelen. Hektor Ammann (Zur Geschichte der Zurzacher Messen, 1929) weist nach, welch großen Anteil am Güter- und Personenverkehr die Wasserstraßen im Mittelalter und bis zur Eisenbahnrevolution leisteten. Im großen Flüssenetz entwickeln sich seine Messen von ersten $\mathrm{Er}$ wähnungen im 13. Jahrhundert bis zur großen Konkurrenz mit den Basler und Zürcher Märkten. Stoffe europäischer Auswahl und Pferdemärkte sind Zurzachs besondere Berühmtheit. Die Reuß verbindet mit Luzern und Flüelen. «Am 9. September (1766) früh um 2 Uhr ist im Hauptdorf (Ursern) an der Matt Feuer ausgebrochen. Starker Wind trieb das Feuer hin und her. Viele Leute aus allen Orten, Deutschland und Frankreich, die Mehrzahl aber von der Zurzacher Messe und aus Italien waren dort über Nacht gelegen und suchten ihre Kaufmannschaft zu salvieren. Es ging ihnen davon nichts verloren. Das Volk aber verlor alles» (Schreiben des Standes Uri vom 1. Oktober 1766).

Der Gotthardverkehr wurde früh winteroffen gehalten. Die Arbeit an der ganzen Gebirgsstrecke, «den Berg zu brechen», den «Schneebruch» zu besorgen, leisteten die Ruttner, italienisch Rottori. Ein enger Weg mußte für Pferde und Ochsenschlitten zur Verfügung gehalten werden. Berlepsch schildert noch 1861 Arbeit und Betrieb. Auch die europäische Attraktion der fünfspännigen Post auf dem «Weltwunder» der neuen Straße von 1830 mußte auf Schlittenbetrieb umgestellt werden. Die Reisenden wurden sehr sorgfältig und sturmsicher verpackt, die Damen fast als Wickelkinder eingebettet und die Schlitten auf der Paßhöhe gedreht, damit in der Abwärtsfahrt «der Kopf wieder höher als die Füße»

\footnotetext{
* An der Vernissage des Kranichdrucks "Der Freiheitsbrief der Schwyzer von Kaiser Friedrich II., Hrsg. Alice Gertrud und Hans Rudolf Bosch-Gwalter.
} 


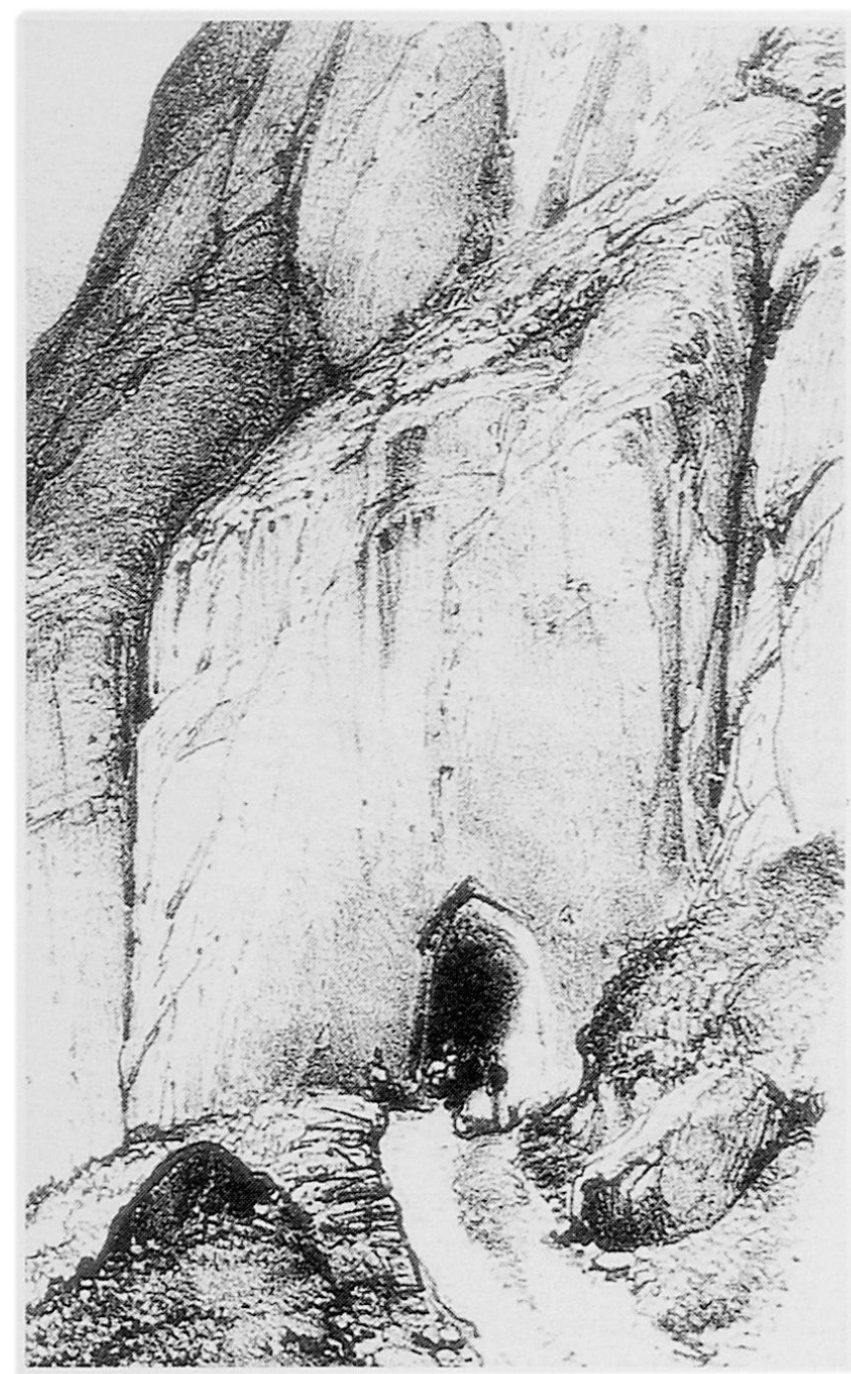

Abb. 2 Urnerloch, wie es Suworow noch angetroffen hat.

lag. («Das Brechen des Berges» nach «alter Ruttnertechnik» wurde im Winter 1984/85 von der Trainkolonne II/42 am tief verschneiten Scalettapaß mit kräftigen, breitbrüstigen Pferden ernstfallmäBig mit Erfolg praktiziert. NZZ 23. /24. März 1985.) In den Gotthardreisebüchern kommt die Schneefühligkeit der Saumpferde oft zu Wort. J. G. Sulzer entgeht 1776 nur durch die hartnäckige Weigerung des Führerpferdes, eine Lawinenschneebrücke über der Reuß (vermutlich Bristenlaui) zu passieren, einem großen Unglück; die Brücke war zu dünn. In einem Gedicht Hölderlins findet sich das Wort: «. . . über den Gotthardt tastet das Roß.»

Die Twärrenbrücke machte fast jährlich Reparaturen nötig und wurde 1707 «durch ein yberschwänklich wasserfluß hinwäg genommen», und die Urner Regierung beschließt, den «bärg durch zuobrächen». Sie beruft dazu den Ingenieur Pietro Morettini von Locarno, der um 1670 als Siebenjähriger «mit einem Trupp von jungen Leuten nach Besançon auswanderte». Das Glück führte ihn in die Obhut und Förderung durch den Festungsbauer
Vauban. Er wurde selber Festungsbaumeister in Genua. (Hans Rudolf Schmid, NZZ 28./29. Juli 1984). Im Sommer 1708 gehen die ersten Säumerzüge durch den «Bergbruch», genannt «Urnerloch», den ersten Straßentunnel von europäischem Rang in den zentralen Alpen. (Das Wort «Tunnel» gab es im Deutschen noch nicht.) Was nun aber das Loch ausgelöst hat an Gotthardbereisung und -beschreibung, ist unvorstellbar. Das heißt, es ist vor allem die Raffung der Erscheinungen, die Schiller in der Parricida-Szene mit den Stichworten «Schrekkensstraße», «Brücke, welche stäubet», «schwarzes Felsentor» und «heitres Tal der Freude» zur Lokkung ins Grauen und in die Erlösung und zur eigentlichen Magie am Gotthard gemacht hat. Und Schillers kreative Geschichtsklitterung 1708 (Loch) und 1308 (Königsmord) ist szenische Steigerung. (Allein über die verbale Fassung der kurzen Landschaftsstrecke besteht bereits privat eine anthologische Textsammlung.) Zur Abtragung der Kosten für den Bergbruch, wo «vorherr außerhalb dem berg nach die twärren brug gstanden» wird ein höherer Zoll als Weg- und «Bruchgeld» verlangt. Im Zusammenhang damit erscheinen auch lange Listen der Zollpreise; z. B. auf erster Zeile: von yödem Saum Käß, wein, ssaltz, koren undt, haber im durchförggen Zuo Ursseren Schl. 1. (Schilling.) Urselerkäs ist europäische Berühmtheit, sein Ruhm fehlt kaum in einem Tagebuch. Kasthofer, der botanische Kenner, sieht seine Qualität durch Kräuter aus der Umgebung von Realp gesichert.

Ungefähr zur Zeit der Urnerlochaktualität erscheint auch der erste geophysikalisch Interessierte am Gotthard: Johann Scheuchzer (1684-1738), der jüngere, zu wenig bekannte Bruder von Johann Jakob Scheuchzer. Bernhard Studer (1863) stellt ihn vor: «Es muß Johann Scheuchzer als der erste schweizerische Geolog genannt werden, der seine Ansichten durch selbständige Beobachtungen in den Alpen zu begründen suchte, als der erste, der auf die fächerförmige Schichtenstellung am Gotthard und auf die wunderbar gekrümmten Schichten am Urner- und Wallensee aufmerksam machte.» Seine erstaunliche, ja verblüffende Urnerseezeichnung (Titelblatt) - man beachte den Axenfels nahe Flüelen, aber auch die westliche Gebirgsflanke wurde von zwei an sich unangefochtenen Koryphäen, Oskar Peschel und Karl Alfred von Zittel ( «Geschichte der Erdkunde» einerseits, «Geschichte der Geologie und Palaeontologie» anderseits), Johann Jakob Scheuchzer zugewiesen. Der Irrtum blieb haften. Mit größter Sorgfalt und genauer Nachweisfolge hat Margrit Koch in der Vierteljahrsschrift der Naturforschenden Gesellschaft Zürich XCVII 1952 die Urheberschaft Johann Scheuchzers gesichert. Unter den vielen, die vom Gotthard angerührt oder gebunden wurden, sei noch der SchweizVerehrer John Ruskin gestreift. Er zeichnete 1835 Hospental - sowohl das Dorf als auch die Land- 


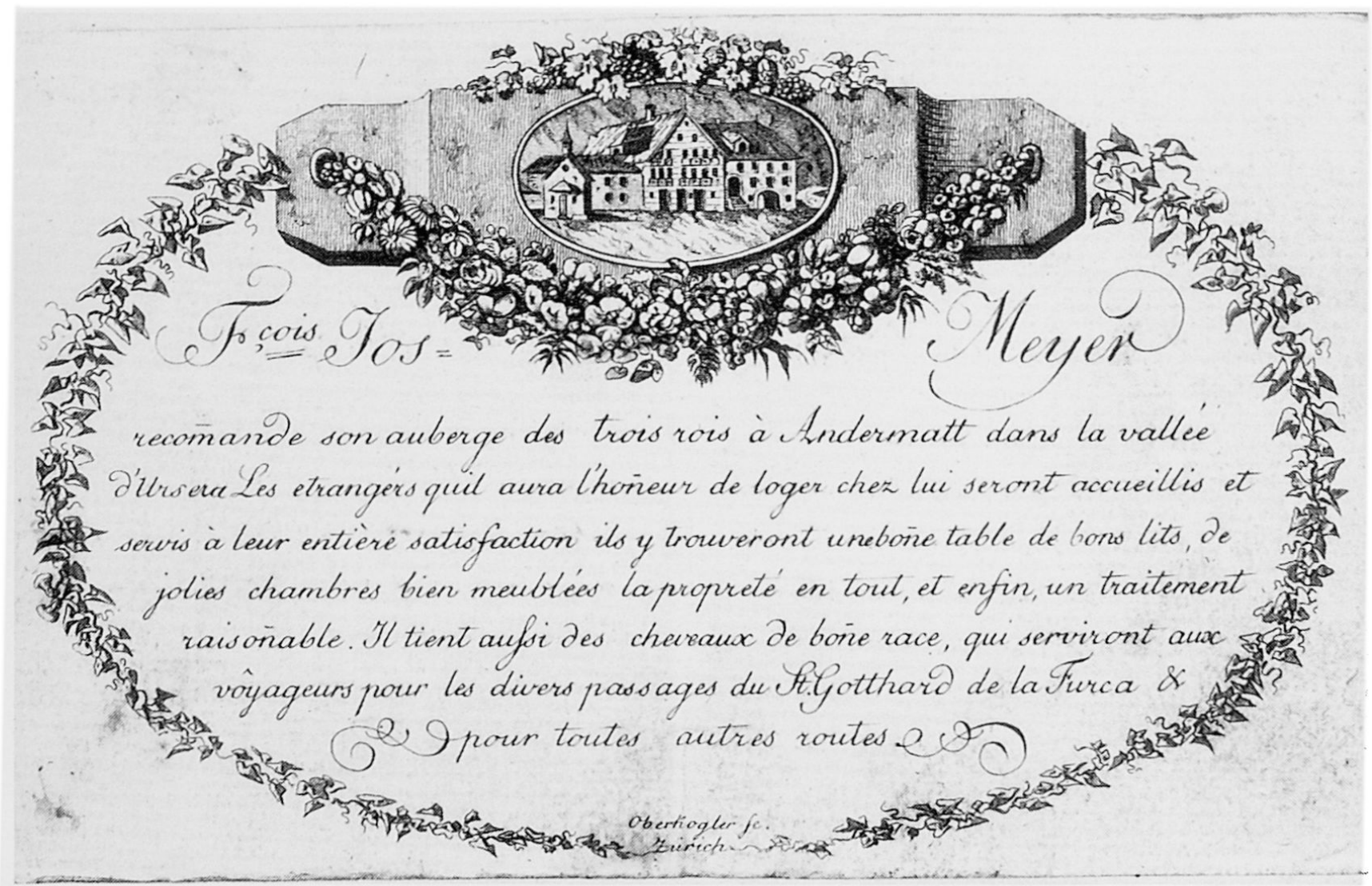

Abb. 3 Reklamekarte des Hotels Drei Könige in Andermatt, üblicherweise französisch, ca. 1850. Talarchivar Myran Meyer, Andermatt, für Druckbewilligung und Hilfe dankbar.

schaft baustilistisch wissend. "Anatomische Arbeit an Bergen», sagt ein Ausstellungskatalog.

Das Datum, das unsere bewaffnete Neutralität mitbegründete, ist nur anzudeuten: der 24./25. September 1799, da das Straßenkreuz von Hospental zum Kampfkreuz wurde. Die Rochadelinie führte Franzosen von der Furka her, die Österreicher vom Oberalp gegeneinander. Auf der NS-Transversale forcierte Suworow von Airolo den von Franzosen hart besetzten $\mathrm{Pa}$, kam bis zum Einnachten ebenfalls nach Ursern. Ob die Franzosen mit der Erfahrung vom Nägelisgrätli in der Nacht den Spitzigrat meistern, ist nicht klar. Ein Versuch der Russen, das Urnerloch zum Inneren Tüfelstal zu umgehen, um die Reuß im Feuer der Franzosen zu durchwaten, scheint nicht zu gelingen, hingegen eine kühne Umgehung am linken Reußufer. Es folgt der berühmte Nahkampf um die Teufelsbrücke. Suworow erreicht schließlich Flüelen, wo keine Schiffe sind. - Der Schweiz wertet das grauenvoll Ganze den Besitz ihres Gotthardzentrums auf.

Ein Erinnerung markierendes Datum war auch der 31. Dezember 1881. Da erfolgte die letzte Postübergabe auf dem Gotthardhospiz und damit die Einstellung des Postkursbetriebes über den $\mathrm{Paß}$. An dem Akt war Isidor Meyer vom renommierten Hotel Dreikönige in Andermatt dabei. Die Inhaber des Hotels waren auch Postpferdehalter. Isidor Meyer
(1860-1944), schließlich auch Landammann, hat die Erinnerung an den Tag des eher bitteren Einzugs des Eisenbahnzeitalters in Ursern später festgehalten. Darin heißt es: «Wie mit einem Schlag schaltete der letzte Dezembertag 1881 die Tradition und Bedeutung des Urserntales im Verkehr mit deutschen und welschen Landen aus und ließ die reiche Erwerbsquelle unserer Bevölkerung versiegen. Wie viele Existenzen hat er auf einmal zerstört und wie viele Ursner zur Auswanderung gezwungen! Postillone, Fuhrleute, Pferdehalter, Weger, Wegknechte, Schmiede, Sattler, Wagner und die vielen Hilfskräfte, die mit dem Transitverkehr in Verbindung standen, wurden plötzlich ihrer Arbeit und ihres Verdienstes beraubt.» Die Einwohnerzahl von Hospental sank von 444 (1870) auf 290 (1900). Manche kehrten wieder zurück. Denn das Leben, wenn auch in anderer Form, kehrte wieder ein in der Talwanne. Andermatt wurde 1890 Waffenplatz. Es gibt den Begriff «Gottharddienst». Es ist ein Dienst im schweizerischen Interesse und in europäischer Neutralitätsverpflichtung, wie er sich mit General Guisans Réduitstrategie bereits bewährt hat.

Das aktuelle Gotthardproblem sind die Basistunnels für Bahn und Straße, wo schweizerisch Humanität und lebensräumliche Menschlichkeit gegen quantitativ orientierte Mobilität kämpfen. Auf daß nicht grenzenlos Zivilisation Kultur überfahre! 